\title{
THE
}

10-16-2011

\section{Single firm product diffusion model for single-function and fusion products}

Yuwen Chen

University of Rhode Island, yuwen@uri.edu

Janice E. Carrillo

Follow this and additional works at: https://digitalcommons.uri.edu/cba_facpubs

Terms of Use

All rights reserved under copyright.

\section{Citation/Publisher Attribution}

Yuwen Chen and Janice E. Carrillo. "Single Firm Product Diffusion Model for Single-Function and Fusion Products" European Journal of Operational Research 214.2 (2011): 232-245. Available online at http://dx.doi.org/10.1016/j.ejor.2011.04.030.

This Article is brought to you for free and open access by the College of Business at DigitalCommons@URI. It has been accepted for inclusion in College of Business Faculty Publications by an authorized administrator of DigitalCommons@URI.For more information, please contact digitalcommons-group@uri.edu. 


\title{
Product Diffusion Model for Single-Function and Fusion Products
}

\author{
Yuwen Chen \\ yuwen@uri.edu \\ College of Business Administration \\ University of Rhode Island \\ Kingston, RI 02881, USA \\ Janice E. Carrillo \\ janice.carrillo@cba.ufl.edu \\ Department of Information Systems \& Operations Management \\ Warrington College of Business Administration \\ University of Florida \\ P.O. Box 117169, Gainesville, FL 32611-7169, USA
}

\begin{abstract}
The prosperity of multifunction products (also referred to as fusion products) has changed the landscape of the marketplace for several electronics products. To illustrate, as fusion products gain popularity in cellular phones and office machines, we observe that single-function products (e.g., stand-alone PDAs and stand-alone scanners) gradually disappear from the market as they are supplanted by fusion products. This paper presents a product diffusion model that captures the diffusion transition from two distinct single-function products into one fusion product. We investigate the optimal launch time of the fusion product in various conditions and conduct a numerical analysis to demonstrate the dynamics among the three products. Similar to previous multi-generation single product diffusion models, we find that the planning horizon, the products' relative profit margin, and substitution effects are important to the launch time decision. However, there are several unique factors that warrant special consideration when a firm introduces a fusion product to the market: the firm's competitive role, buyer consolidation of purchases to a multifunction product, the fusion technology and the age of current single-function products. Key words: multifunction products, fusion products, product diffusion
\end{abstract}




\section{Introduction}

\subsection{Examples and Motivation from Industry}

The prosperity of multifunction products (also referred to as fusion products) has changed the landscape of the marketplace for several electronics products. After the launch of a fusion product (FP) into the marketplace, the sales of some single-function products (SPs) start to decline. Examples from the popular business press illustrating this phenomenon are numerous. To demonstrate, all-in-one products with printing, copying, scanning and fax functions have become ubiquitous in the office machine market. Based on Gartner's Latin America market report, in the first quarter of 2003, the single-function inkjet printer sales declined $5 \%$ from the same period of 2002 , while the sales of multi-function inkjet printer increased $176 \%$ in the same time, (Gartner, 2003).

In 2006, IDC's report on the worldwide handheld ${ }^{1}$ market finds that shipments were down $22.3 \%$ in the first quarter of 2006 from the same quarter the prior year. Furthermore, this yearover-year decline has continued for the last nine consecutive quarters, (cellular-news.com a, 2006). According to this report, reasons for the decline of the single-function hand-held devices include the following: some manufacturers are exiting from the market altogether, the remaining manufacturers are switching production to converged mobile devices (multifunction cellular phones), and the converged mobile devices are becoming increasingly popular in the marketplace, (Palminfocenter.com, 2006). Many other market analysts predict that a multi-function personal device will eventually replace many personal electronic products. In Gartner's report ${ }^{2}, 53 \%$ of PDAs have some sort of integrated cellular capability (Ferguson, 2006). One reason for the shift in the PDA market cited in this report is that the wireless service carriers essentially subsidize these devices and drive the price of PDA phones down in exchange for monthly data service fees (cellular-news.com b, cellular-news.com c, 2006).

For wireless communication, consumers find more cell phones with extra functions, such as digital camera, PDA, digital music and video player. Consequently, not many cell phone models now are equipped with only a basic cell phone function. The research study released by ABI Research in 2005 (ABI Research, 2005) projects that the shipment of camera phones will exceed the shipment of non-camera phones worldwide. Multi-functionality and inexpensive prices continue to boost the fusion product camera phone's popularity. This report also forecasts that the camera phone will eventually replace a portion of the digital camera market. The fact that many digital camera makers now only offer models with resolution of seven mega-pixel or above is evidence of

\footnotetext{
${ }^{1}$ In IDC's report, the term Handheld is used equivalently as palm-size personal digital assistant (PDA) in this report. Any handheld equipped with cellular function is defined as a smartphone.

${ }^{2}$ In Gartner's report, the boundary of PDA includes the single-function PDA and the cellular-equipped PDA and the PDA is seen as a data-centric (voice second) device. The smartphone, which offers all the attributes of PDA, is a voice-centric (data second) device.
} 
the camera phone's encroachment in the camera industry. The low-end digital camera market has shrunk significantly from the popularity of the camera phone.

\subsection{Motivation of Key Issues}

A diffusion model is useful in this perspective to analyze the transition in the marketplace from single-function to multi-function products. The diffusion of a product is described as the projection of the sales rate and cumulative sales throughout the product's life cycle. Early in a product's life cycle, the diffusion of an innovation first reaches the early adopters. Gradually these adoptions reach the rest of a social system through interpersonal communication with early adopters. Assuming the adoption pattern is a normal distribution, Roger (2003) categorizes the adopters into five groups based on the timing of their purchase: innovators, early adopters, early majorities, late majorities, and laggards.

The phenomenon above is similar to the diffusion process of several generations of products, but there is a major difference: the research on the diffusion process of several generations only focuses on one product category, but fusion products influence the product diffusion across several product categories. For example, the camera phone has changed the market landscape in the cellular phone and the digital camera. The diffusion model which treats the FP as a new generation of an SP might not be sufficient to capture all of the dynamics brought by the FP. In addition, how well the fusion product consolidates its component products has a strong influence on the adoption of the FP. Thus, using a multi-generation diffusion model, the diffusion paths derived from two SPs might be very different.

Moreover, if there is synergy among some SPs then fusing them together into an FP is appealing to the market. For the consumer, the benefits of the FP may include space saving, lower total cost, and function synchronization. For the firm, a key benefit of launching FPs may be manufacturing cost savings. A printer, a copier, a scanner and a fax machine can all share the same platform and interface which makes a multifunction office machine cost just slightly higher than a singlefunction printer. The cost savings can make it possible for the FP to be priced lower than the sum of all SPs. The early generations of multifunction office machines had high profit margins due to manufacturing cost savings and strong demand.

Finally, the competitive role of the FP's manufacturer in the market is also significant. The FP's manufacturer can be a new entrant or an incumbent of one or more current SPs. In the office machine market, the manufacturer of the scanner and the printer is also the manufacturer of the all-in-one office machine. But in the personal portable device market, the first PDA phone was introduced by Palm, which is a PDA manufacturer. In contrast, the successful iPhone was introduced by Apple, which is the leader in the digital music player market. We will also analyze the impact of the competitive role of the FP's manufacturer on the FP's launch decision and on 
the diffusion processes.

Note that we do not explicitly model competition between manufacturers in this paper. Instead, we capture the competitive role of the manufacturer in terms of its current product offerings. Specifically, we develop a model for a single firm based on the Bass model which captures the intertemporal dynamics of product diffusion in the market. A primary motivation for this approach is the complexity of the model. However, we believe that a dynamic game theoretic model has the potential to make a positive contribution to this literature in the future.

In general, product diffusion research focuses on the diffusion within a product category. Current diffusion models do not capture the transition from single-function products to fusion products. In this paper, we construct a new product diffusion model that is as parsimonious as possible to capture the diffusion process of the FP and their interactions with the diffusion processes for the SPs. After the launch of the FP, the FP starts encroaching on the demand of the SPs and changes their diffusion paths. We are interested in identifying the optimal launch time decision for the fusion product and the factors that influence the decision. The following questions related to fusion product diffusion still need to be answered: (a) How long will it take to penetrate to a certain level of market acceptance? (b) When should the firm introduce the fusion product? (c) How does the manufacturer speed up the penetration of a new fusion product? (d) How do development costs and technology maturity influence this decision? (e) How does the role of the manufacturer (i.e. incumbent or new entrant) influence these decisions?

In the next section, we will review the literature related to the topic of product diffusion and highlight those most related to our model.

\section{Literature Review}

In this section, we first review the literature from business and technology journals concerning the benefits and drawbacks of FPs from the consumer's perspective. We also summarize the academic literature explicitly addressing FPs. Then we introduce the literature on Bass diffusion models and emphasize on those most relevant to FPs.

\subsection{Multi-Function Products}

In recent years, business and technology journals have published several reports on the nature of FPs. These reports address key issues such as "how to select the right FP" (Avery, 2003), "the manufacturer's strategy" (Avery 2004), "new market trend" (Anonymous, 2005; Gilroy, 2004; Meyers, 2004), "product design critique" (Harbaugh, 1998), and "multi-function product review" (Magid, 1998; Rysavy, 2004; Breeden and de Soto, 2000).

There are several key findings contained in these business and technology reports associated with 
FPs. First, as the prices of FPs decline, the FPs are more affordable to consumers. Evidence also shows that the early versions of FPs often have compromised the quality of some of its functions (Harbaugh, 1998; Schonfeld, 2004). Several articles (Avery, 2004; Magid, 1998) show that the demand for FPs starts to grow when the quality of FPs became more acceptable. As technology advances continue, fusion products will emerge and prosper in more product fields. Another key benefit to consumers of an FP is "less machine count" (total number of single-function devices). Finally, FPs normally work well in one or two functions but not all of them, and the consumers should evaluate what level of performance they require and how much they are willing to pay.

Though many business and technology journals have paid attention to the proliferation of FPs (Anonymous, 1997; Cullen, 1999; Harbaugh, 1998; Magid, 1998; Meyers, 2004; Schonfeld, 2004), there are still very few academic, theoretical papers discussing this phenomenon. Thompson, Hamilton, and Rust (2005) investigate consumer's feature fatigue while purchasing the multi-feature products. They find that most consumers do perceive that "more is better" before they really buy and use the FPs. It was observed that adding too many features decreases the usability of the FP to the consumers and they experience buyer's regret.

Chen, Vakharia and Alptekinoğlu (2008a) study the optimal product portfolio for a firm that offers two distinct SPs and one FP. They find that when the profit margin of the FP increases (from either increasing market potential or decreasing manufacturing cost), the firm should first phase out the SP with low profit margin and high substitution with the FP. Chen, Vakharia, Carrillo, and Sin (2009) focus on identifying the optimal product portfolio including both SPs and FPs when a large number of FPs are technologically feasible. However, these models are static and do not consider the dynamic transition from single-function to multi-function products in the market. If we analyze the problem using the traditional product life cycle, the changes of market transition from SP to FP occur gradually. The launch of FPs is often several years behind their precedents SPs. As the FPs gain popularity, the sales of the SPs peaks prematurely and the FPs become more popular.

We also address the competitive role of the supplier of the FP. A key question influencing the firm's decision to introduce an FP concerns its current product offerings of SPs in the marketplace. Early literature in the strategy area finds that incumbent firms are believed to have a competitive advantage when introducing new products (Lieberman, and Montgomery, 1988). Three primary sources of advantages for the incumbents are: (1) technological leadership, (2) preemption of assets, and (3) buyer switching cost. Schmidt and Porteus (2000) propose a model that a firm can sustain its leadership through cost and innovative competence. Min, Kalwani and Robinson (2006) find that the pioneer has higher risk of failure in the markets started by a radically different new product. In markets started by incremental innovation, the pioneer's risk is much lower. 


\subsection{Product Diffusion}

Bass (1969) proposes an S-shape diffusion pattern as a new product is introduced to the market then diffuses gradually to the whole market. The S-shape is defined as the cumulative sales of the product. The sales rate over time is usually a bell shaped curve which includes several stages from birth, growth, peak, to decline. The diffusion pattern has several important components: total market potential $(m)$, innovation coefficient $(\phi)$ and imitation coefficient $(\psi)$ (Mahajan, Muller and Wind, 2000). As a new product is launched, it first reaches some innovative adopters in the whole market. As time goes by, the product's prevalence spreads from the 'neighborhood' of the innovative adopters as the other adopters imitate and become the followers. The Bass model is known for its robustness in characterizing the diffusion process without incorporating decision variables.

Mahajan et al. (2000) point out that new product diffusion models have two different strategic decisions: prelaunch/launch decisions vs. postlaunch decisions. In postlaunch diffusion research, Mahajan et al. (2000) illustrate six applications: timing of successive generations, capacity decisions with product diffusion (Jain et al., 1991; Ho, Savin and Terwiesch, 2002), determining the market value on anticipated penetration (Kim, Mahajan and Srivastava, 1995), market saturation assessment and expansion opportunity for the retailers (Mahajan, Sharma, and Kerin, 1988), estimation of lost sales due to pirated sales (Givon, Mahajan and Muller, 1995), and lost sales due to patent infringements (Mahajan, Sharma, and Buzzell, 1993).

There are many papers related to product or technology diffusion. For a summary of the existing literature, we refer the reader to Mahajan, Muller and Bass (1990) and also to the book edited by Mahajan et al. (2000). Here, we review several papers that are most relevant to our fusionproduct diffusion problem and related to the diffusion and substitution between several generations of technology or product. Note that most of the literature in this area is limited to addressing successive generations of products in a single product category.

Norton and Bass (1987) construct a diffusion model for repeat-purchase products where the new technology brings successive generations of innovation. Different from previous market-share substitution models (e.g., Fisher and Pry, 1971), their diffusion model has the ability to estimate a market potential and forecast the demand trajectory. By fitting their model with data from the DRAM and SRAM markets, they find that once the new generation is introduced, the sales of the older generation begins to decline.

Wilson and Norton (1989) investigate the optimal time to introduce new generations of a durable product. They find that several factors primarily influence the result: the substitution effect and diffusion between two products, the relative margins, and the planning horizon of the firm. This model does not consider market growth over time in a dynamic scenario. They admit that a static 
analysis and a dynamic analysis may obtain different conclusions.

Mahajan and Muller (1996) extend the Bass diffusion model that captures the substitution of successive generations of products. Under some assumptions that simplify the complexity of their diffusion model, they find that the new generation should be either introduced as soon as it is available or at the maturity stage of the previous generation. This "now or at-peak" decision depends on the gross profit margins, the diffusion and substitution parameters, the relative size of market potentials, and the discount factor. They apply their model to the IBM mainframe market and show that, in hindsight, IBM probably introduced new generations to the market too late. Note that Mahajan and Muller (1996) characterize the demand function in a different manner than Wilson and Norton (1989). Our model is more similar to Mahajan and Muller (1996).

Another important empirical model of the diffusion of telecommunication products is conducted by Kim, Chang and Shocker (2000) in Hong Kong and South Korea. Their dynamic market growth model captures both technology substitution as well as inter-product category effects. Their diffusion fitting results show that their model can make a better prediction than the general Bass model. However, they also point out that their empirical model cannot answer strategic questions such as the optimal launch time of a new product.

Several diffusion papers discuss the relationship of diffusion among several products. Putsis (1989) applies the diffusion model into the diffusion of stand alone TVs and VCRs by considering both cross-product demand and supply issues. Van den Bulte (2000) investigates the diffusion speed of 31 electrical household durables in the United States from 1923 to 1996. The results show a statistically significant acceleration on the diffusion speed. Talukdar, Sudhir, and Ainslie (2002) find that the diffusion of a product introduced earlier in one country is useful to explain the diffusion coefficients in other countries, while the past experience of one product is more useful to estimate the penetration level of another product in the same country.

Several papers focus on estimation of diffusion parameters. Norton and Bass (1987) assume that the innovation and imitation parameters over generations are the same. They admit that this is a strong assumption that fits the empirical data. Mahajan and Muller (1996) assume the same innovative parameter but a different imitation parameter. Sohn and Ahn (2003) use Monte Carlo simulation to find the impact of initial factors on the diffusion for new information technology. Also, Pae and Lehmann (2003) investigate the correlation between the inter-generation time and the diffusion data fitting. They report that the later generation seems to have smaller initial sales (smaller innovation parameter) but a faster rate of growth (larger imitation parameter) based on their 30 pairs of two-generation products. They reach this conclusion from an ordinary least squares regression analysis which obtains the changes in parameter values $(\phi$ and $\psi$ ) between generations. However, Van den Bulte (2004) cautions that Pae and Lehmann's (2003) finding may be a methods artifact since several simulation studies (van den Bulte and Lilian, 1997; Bemmaor and Lee, 2002) 
have similar findings.

\section{Model}

\subsection{Diffusion of Single-Function Products}

For our model, we focus on the situation where two SPs are currently in the market, and one FP has become technologically achievable. The notation used in this paper is listed in Table 1. We assume that the market currently has two distinct SPs, denoted as 1 and 2, respectively. Let $m_{i}$ be the total adopters (or market size) for product $i(i=1,2)$. We assume that, without other interference, the demand trajectory for $\mathrm{SP} i$ is exogenous and influenced by two factors: the independent innovation coefficient $\phi_{i}$ and the word-of-mouth (imitative) coefficient $\psi_{i}$. Note that production constraints and uncertainty of demand are not modeled in this paper. As a result, the firm always produces exactly the amount of the demand; consequently, the sales rate at a particular time equals the demand. According to the Bass Model (1969), the sales rate for product $i$ at time $t$ can be formulated as follows.

$$
s_{i}(t)=\frac{m_{i}\left(1+a_{i}\right) b_{i} e^{b_{i} t}}{\left(a_{i}+e^{b_{i} t}\right)^{2}} \quad i=1,2,
$$

where $a_{i}=\frac{\psi_{i}}{\phi_{i}}$ and $b_{i}=\psi_{i}+\phi_{i}$. Note that the equation assumes the current time is at $t=0$. However, when we analyze the sales trajectories for the existing SPs, the products have normally been in the market for some time. We assume that when the fusion technology is achievable, the ages for SP 1 and SP 2 are are $t_{1}$ and $t_{2}$ periods old, respectively. Thus, we allow for the possibility that the two SPs are at different stages in their respective life cycles. For example, the cellular

phone for the consumer market in the U.S. was launched in the early 80's, but the first successful PDA for the consumer market was launched in 1997 by Palm.

\section{Insert Tables 1 here}

Given that the SPs have been in the market for some time, the sales rate at time $t$ for product $i$ should be revised as:

$$
s_{i}(t)=\frac{m_{i}\left(1+a_{i}\right) b_{i} e^{b_{i}\left(t+t_{i}\right)}}{\left(a_{i}+e^{b_{i}\left(t+t_{i}\right)}\right)^{2}} \quad i=1,2
$$

Let $t=0$ be the present time. For $t>0, s_{i}(t)$ represents the sales rate of product $i$ at time $t$. The diffusions of two SPs are independent and deterministic when there is no interference from other factors. However, the fusion technology changes the dynamics of the product diffusion. 
At the present time $(t=0)$, the manufacturer of the FP owns the fusion technology and is considering entering into the FP market. From the discussion in the literature, the manufacturer of the FP can either be a new entrant or an incumbent. This model investigates the optimal launch time of the fusion product when the fusion product manufacturer is in one of the following scenarios: (I) a total new entrant, (II) a manufacturer of one SP, and (III) the manufacturer of two SPs. In this section, our analysis for scenario II assumes that the firm is the manufacturer of both SP 1 and FP. The scenario that the firm is the manufacturer of both SP 2 and FP is symmetric to scenario II and is thus omitted. However, in the numerical analysis, we separate the former and the latter situations as II.1 and II.2 scenarios, respectively.

\subsection{Development Cost for the Fusion Product}

While the technology for the FP becomes available at time $t=0$, we assume that it is not necessarily mature yet. Specifically, the manufacturer may choose to make investments in further development of the FP technology prior to releasing the FP to the market.

We assume that at $t=0$, the manufacturer of the FP can choose to launch a fusion product with a fixed development cost $v_{0}$, or launch the fusion product later. A classic trade-off that many firms face concerns the interplay between time-to-market, product quality and development cost (Bayus, 1997). An early-launched new product will grab the market sooner, but this strategy also contains the risks of high development cost, lower quality from poor design, and possible failure of product transition when not enough adopters purchase the product. A U-shaped development cost function is proposed by Smith and Reinertsen (1991), Gupta et al. (1992), and Murmann (1994) and Bayus (1997). If the firm wants to speed up product development and to launch the FP immediately, the firm needs to invest more in product development. If a new product development project take too long to finish, then the development cost can be trimmed by reducing the development time. Let $t_{m}$ to be the time with minimal development cost of this fusion technology; hence, $t_{m}$ represent the technology cost maturity. Similar to Bayus (1997), we assume the development cost $v$ of the FP launched at time $\tau$ follows a U-shape as described below.

$$
\begin{aligned}
v(\tau) & =v_{0}-\alpha \tau t_{m}+\alpha \tau^{2} / 2=v_{0}-\alpha t_{m}^{2} / 2+\alpha\left(\tau-t_{m}\right)^{2} / 2 \quad \tau \geq 0 \\
\dot{v} & =\frac{d v}{d \tau}=\alpha\left(\tau-t_{m}\right)
\end{aligned}
$$

The development cost curve and the first derivative of development cost with respect to time are shown in Figure 1. The first derivative of the development cost is decreasing (increasing) if the FP is launched before (after) $t_{m}$, and the minimal total development cost $\left(v_{0}-\alpha t_{m}^{2} / 2\right)$, occurring when the FP is launched at $t_{m}$, is assumed to be positive. The coefficient $\alpha$ indicates the convex 
increasing rate of the development cost when the launch time deviates from $t_{m}$. The second part of Figure 1 shows that $\dot{v}$ is increasing in time. Note that this is a major difference from the model in Mahajan and Muller (1996). They do not consider the impact of development cost, which may vary with the launch time of the new generation.

Insert Figure 1 here

\subsection{After the Availability of the Fusion Product}

The launch of the FP disturbs the diffusions of SPs 1 and 2. The substitution between the FP and the SPs is similar to the substitution between products of different generations. To make the model tractable, we assume that there is only one generation of FP considered. The FP made available by the fusion technology is indexed as product 3 and is associated with additional adopters of population size $m_{3}$. The composition of $m_{3}$ includes (1) the consumers who are not included in $m_{1}$ or $m_{2}$, and (2) the consumers who had purchased one or two SPs before the launch of the FP and will 'upgrade' to purchase the FP. Van den Bulte and Lilien (1997) point out the $m_{3}$ can come from population growth.

Let $\tau$ be a decision variable which is the launch time of the FP. The launch time is critical to the firm because it often impacts on many aspects of diffusion. First, to achieve an earlier launch time, the firm often needs to invest more in product development. Second, the earlier the launch of the new product, the more potential buyers of the old products may switch to buy the new product. Third, the firm's strategic concerns for the launch time may depend on its current product portfolio (scenario I, II or III).

To simplify the model's complexity, we assume only the development cost of product introduction is affected by the launch time decision. Similar to existing literature on multi-generation diffusion models, we assume that the launch time of the FP will not impact the values of other parameters. The total adopters $m_{3}$, the profit margin of FP, the switching rate, and the diffusion parameters are independent of the launch time.

However, the launch of FP 3 provides the future adopters in $m_{1}$ and $m_{2}$ an option. Norton and Bass (1987) and Mahajan and Muller (1996) both assume that (a) a new generation product expands the market and (b) the substitution effect is captured by some proportion of consumers for the old generation product that will switch to a new version of the product. We have a similar assumption and we allow different switching rates for the two SPs. Normally, the SP with a higher switching rate has a higher similarity to the FP. For example, an all-in-one printer works pretty well as a printer and less well as a scanner. For the future buyers of the the single-function printer, the consumers normally buy either a single-function printer or a multi-function printer. Specifically, it is more likely that consumers for the printer will switch to the fusion product. Conversely, the 
future buyers of a single-function scanner are less likely to purchase the fusion product since the scanning in the fusion product is the secondary feature.

Note that many consumers are often simultaneously included as future buyers for multiple distinct SPs. For example, many consumers have requirements for both a printer and a scanner. When an FP is launched and attracts these future buyers of SPs, some buyers may eventually "buy one FP instead of two distinct SPs". As a result, in our model, we have to avoid repeatedly counting the switch of SP future buyers to the FP. It is very difficult to determine exactly how many future buyers from SPs switch to buy the FP. To simplify the estimation of the switch, we assume that a fraction $w(0 \leq w \leq 1)$ of the total switches from both SPs is contributing to increasing the demand for FP 3.

Now we construct the sales trajectories after the launch of the FP. To simplify the notation, we drop the time argument in related variables when possible. Let $s_{i}^{n}$ be the sales of product $i$ considering the consumer switch after the launch of the FP, then

$$
\begin{aligned}
& s_{i}^{n}=s_{i}\left(1-R_{i}\right) \quad \text { for } i=1,2, \\
& s_{3}^{n}=s_{3}^{m_{3}}+w\left(s_{1} R_{1}+s_{2} R_{2}\right)
\end{aligned}
$$

where $0 \leq R_{i}<1(i=1,2)$ represents the switching rate of the demand of SP $i$ switching to the FP, and $s_{3}^{m_{3}}=\frac{m_{3}\left(1+a_{3}\right) b_{3} e^{b_{3}(t-\tau)}}{\left(a_{3}+e^{b_{3}(t-\tau)}\right)^{2}}$ indicates the future adopters of FP 3 from $m_{3}$ (Bass, 1969). Note that Mahajan and Muller (1996) use a similar form to capture the switching from the old to the new generation. The expression $R_{i} s_{i}(i=1,2)$ represents the adopters that switch from SP $i$. However, due to overlapping customer needs, only $w\left(s_{1} R_{1}+s_{2} R_{2}\right)$ will purchase the FP.

\subsection{Objectives and Analysis}

Let $d_{1}, d_{2}$ and $d_{3}$ be the unit profit of products 1,2 , and 3 , respectively. We do not consider the discount factor in our objective function for two reasons. First, it is difficult to identify when and how much the development cost occurs at any point of time since this cost occurs before the launch time. Second, omitting the discount factor allows us to identify other factors influencing the optimal solution. The firm's goal is to find the optimal launch time which maximizes their profit.

We previously mentioned the manufacturer of the FP can be either an incumbent or a new entrant. In this model, the FP's manufacturer is will be one of three following scenarios (I) a total new entrant, (II) a manufacturer of one SP, and (III) the manufacturer of two SPs. 
The objective functions of the firm under the three scenarios are as follows.

$$
\begin{aligned}
\max _{\tau} \Pi_{I} & =\int_{\tau}^{T_{3}}\left[d_{3} s_{3}^{n}\right] d t-v(\tau) \\
\max _{\tau} \Pi_{I I} & =\int_{0}^{\tau}\left[d_{1} s_{1}\right] d t+\int_{\tau}^{T_{3}}\left[d_{1} s_{1}^{n}+d_{3} s_{3}^{n}\right] d t-v(\tau) \\
\max _{\tau} \Pi_{I I I} & =\int_{0}^{\tau}\left[d_{1} s_{1}+d_{2} s_{2}\right] d t+\int_{\tau}^{T_{3}}\left[d_{1} s_{1}^{n}+d_{2} s_{2}^{n}+d_{3} s_{3}^{n}\right] d t-v(\tau)
\end{aligned}
$$

The firm in scenario I is a new entrant and is concerned chiefly with the profit of the FP after the launch. The firm in scenario II needs to evaluate the total profit from SP 1 before the FP's launch and the total profit of SP 1 and FP 3 after the launch. The objective function of the firm in scenario III includes the profit of SPs 1 and 2 before the FP's launch and the total profit of all products after the FP's launch.

Taking the first derivative of the profit functions with respect to $\tau$, we obtain

$$
\begin{aligned}
\frac{d \Pi_{I}}{d \tau} & =-d_{3}\left[s_{3}^{m_{3}}\left(T_{3}\right)+w\left(R_{1} s_{1}(\tau)+R_{2} s_{2}(\tau)\right)\right]-\alpha\left(\tau-t_{m}\right) \\
\frac{d \Pi_{I I}}{d \tau} & =d_{1} R_{1} s_{1}(\tau)-d_{3}\left[s_{3}^{m_{3}}\left(T_{3}\right)+w\left(R_{1} s_{1}(\tau)+R_{2} s_{2}(\tau)\right)\right]-\alpha\left(\tau-t_{m}\right) \\
\frac{d \Pi_{I I I}}{d \tau} & =d_{1} R_{1} s_{1}(\tau)+d_{2} R_{2} s_{2}(\tau)-d_{3}\left[s_{3}^{m_{3}}\left(T_{3}\right)+w\left(R_{1} s_{1}(\tau)+R_{2} s_{2}(\tau)\right)\right]-\alpha\left(\tau-t_{m}\right)
\end{aligned}
$$

By setting the first order conditions zero, we obtain:

$$
\begin{aligned}
-\alpha\left(\tau-t_{m}\right) & =d_{3}\left[s_{3}^{m_{3}}\left(T_{3}\right)+w\left(R_{1} s_{1}(\tau)+R_{2} s_{2}(\tau)\right)\right] \\
d_{1} R_{1} s_{1}(\tau)-\alpha\left(\tau-t_{m}\right) & =d_{3}\left[s_{3}^{m_{3}}\left(T_{3}\right)+w\left(R_{1} s_{1}(\tau)+R_{2} s_{2}(\tau)\right)\right] \\
d_{1} R_{1} s_{1}(\tau)+d_{2} R_{2} s_{2}(\tau)-\alpha\left(\tau-t_{m}\right) & =d_{3}\left[s_{3}^{m_{3}}\left(T_{3}\right)+w\left(R_{1} s_{1}(\tau)+R_{2} s_{2}(\tau)\right)\right]
\end{aligned}
$$

Unfortunately, the optimal launch times solved by the first order conditions above are in a complicated form, such that no closed-form solution can be obtained. Wilson and Norton (1989) mention the optimal launch time solution can be found by taking the derivative of the profit function with respect to the launch time decision and finding the value of the launch time when the derivative vanishes. However, they also point out that the value of the optimal launch time is "sufficiently complicated that it is challenging to get insight about its implication." Mahajan and Muller (1996) use optimal control theory to investigate the optimality condition, but do not obtain a closed form solution.

The second derivatives of the profit functions with respect to $\tau$ are presented below. Let $\dot{s}_{i}$ be defined as the first derivative of the sales for product $i$ with respect to time. Since $\dot{s}_{1}, \dot{s}_{2}$, and $\dot{s}_{3}^{m_{3}}$ can be either positive or negative depending on the increasing or decreasing sales rates, the global 
concavity of the profit function cannot be guaranteed.

$$
\begin{aligned}
\frac{d^{2} \Pi_{I}}{d \tau^{2}} & =d_{3}\left[\dot{s}_{3}^{m_{3}}\left(T_{3}\right)+w\left(R_{1} \dot{s}_{1}(\tau)+R_{2} \dot{s}_{2}(\tau)\right)\right]-\alpha \\
\frac{d^{2} \Pi_{I I}}{d \tau^{2}} & =-d_{1} R_{1} \dot{s}_{1}(\tau)+d_{3}\left[\dot{s}_{3}^{m_{3}}\left(T_{3}\right)+w\left(R_{1} \dot{s}_{1}(\tau)+R_{2} \dot{s}_{2}(\tau)\right)\right]-\alpha \\
\frac{d^{2} \Pi_{I I I}}{d \tau^{2}} & =-d_{1} R_{1} \dot{s}_{1}(\tau)-d_{2} R_{2} \dot{s}_{2}(\tau)+d_{3}\left[\dot{s}_{3}^{m_{3}}\left(T_{3}\right)+w\left(R_{1} \dot{s}_{1}(\tau)+R_{2} \dot{s}_{2}(\tau)\right)\right]-\alpha
\end{aligned}
$$

Given that the first derivatives of sales rate are positive before the sales peak and negative after the sales peak, the second order conditions show that the profit function is generally concave when $\tau$ is small and the sales from the SPs are still increasing. But in some cases, at some point after the sales peaks of the SP, the profit function starts to become convex decreasing as $\tau$ increases. This case is more likely to occur in scenario I than scenarios II and III since the magnitude of negative terms in scenario I is smaller. In our numerical examples, some cases show the profit functions are strictly concave in the planning horizon.

Even though the optimal launch time cannot be explicitly derived, we can still gain some insights from equations 6 to 8. The right hand sides of the FOCs represent the marginal profit from the FP, and the left hand sides of the FOCs equal the marginal development cost plus the marginal loss of profit from the $\mathrm{SP}(\mathrm{s})$. For example, in scenario I, the optimal launch time only occurs between now and the technology cost maturity $\left(t_{m}\right)$ since the RHS is always positive. The managerial interpretation is that the firm should launch the FP at the moment that the marginal profit of the FP equals to the marginal cost from the development. The marginal profit for launching the FP equals the FP's profit margin times the sum of FP's sales at the end of planning horizon and the switched demand from SPs at the launch time. Note that the sales of FP at the end of planning horizon comes into the equation. Given a certain planning horizon, the launch time of the FP will decide the cumulative sales level of the FP. The sales of the FP from $m_{3}$ always start from zero; as a result, the sales of the FP from $m_{3}$ at the ending becomes part of the marginal profit. This result implies that the length of planning horizon will impact on the decision of launch time. Similarly, in scenario II (III), the optimal launch time occurs when the marginal profit of the FP equals to the sum of the marginal cost from the development and the lost profit(s) from $\mathrm{SP}(\mathrm{s}) 1$ (and 2).

From the composition of $\frac{d \Pi_{I}}{d \tau}, \frac{d \Pi_{I I}}{d \tau}$ and $\frac{d \Pi_{I I I}}{d \tau}$, we notice that $\frac{d \Pi_{I I I}}{d \tau}>\frac{d \Pi_{I I}}{d \tau}>\frac{d \Pi_{I}}{d \tau}$ when all parameter values remain the same for the three scenarios and the concavity of the second order conditions holds. From the structure of the FOCs in three scenarios, we can conclude that the new entrant (scenario I) should choose a launch time which is earlier than the firm in scenario II, which again should choose a launch time earlier than the firm in scenario III. This is a reasonable outcome since a new entrant has no concern about the cannibalization of the FP to the SPs, and the firm in scenario III must evaluate the cannibalization of the FP to two SPs. When the firm of 
the FP is also the supplier of SPs, extra profit from the FP sales comes with some sales loss from the SPs. As a result, the opportunity cost of an earlier launch is higher for the firm in scenario III than the firms in I and II when other conditions remain the same.

When $\frac{d \Pi_{i}}{d \tau}>0$ for $i \in\{I, I I, I I I\}$ and for any $\tau \in\left[0, T_{3}\right]$, this indicates that the FP is very profitable compared to the development cost and the profit loss from $\mathrm{SP}(\mathrm{s})$, and the firm of scenario $i$ should introduce the FP immediately. Similarly, when $\frac{d \Pi_{i}}{d \tau}<0$ for $\tau \in\left[0, T_{3}\right]$, the the firm of scenario $i$ should not introduce the fusion product during this planning horizon since the possible lost profit from SPs plus the development cost is higher than the profit from the FP. Note that the magnitude of $\alpha$, which represents the convex increasing rate of total development cost, is included in the necessary conditions. When $\alpha$ is large, the total development cost will increase significantly when the launch time of the FP deviates from $t_{m}$. In this circumstance, the optimal launch time of the FP will likely be more 'centered' to $t_{m}$. If the development cost over time is a flatter curve, which has a smaller $\alpha$, then the optimal launch time of the FP will be earlier (later) compared to the case with a steeper development cost curve. That is, the optimal launch time of the FP is more likely to deviate from $t_{m}$ when the development cost over time is a flatter curve.

From the analysis above, we know that no closed-form analytical solution can be obtained using the general functional forms for diffusion and costs. In the next section, we exemplify many sets of numerical examples to study the optimal FP launch time.

\section{Numerical Analysis}

In this section, we use different sets of parameter values to investigate the diffusion problem of two SPs and one $\mathrm{FP}^{3}$. We investigate the impact on the the optimal solution of the examples by changing one parameter value for all cases. However, across all examples, we select one base case as the basis for comparison. The parameters we use for the examples can be categorized into four groups. The first group addresses the characteristics of the fusion product: the diffusion speed, the relative profit margin, and the new future adopters of the fusion product. The second group is related to the technology and the development cost: development cost curve and the technology cost maturity. The third group contains time related factors: the age of the SP and the planning horizon. The fourth group concerns the SP related parameters: their relative size, the substitution effects of the FP on the SPs, and the overlap degree in the future adopters of two SPs.

In the real world, the SPs may have different market sizes and profit margins. To reflect this situation, in our numerical examples, we let SP 2 have a larger market size $\left(m_{2}>m_{1}\right)$ and a higher

\footnotetext{
${ }^{3}$ There is no reliable market data available for fitting the diffusion model with SPs and FPs. First, it is lack of clear-cut boundaries between FPs and SPs. Second, the market survey firms use various definitions of categorization of products (see section 1). For example, a PDA phone which is categorized in the PDA shipment by a market research firm can be included in cell phone sales in another firm. These two reasons make the industrial data unreliable for fitting the product diffusions.
} 
unit profit $\left(d_{2}>d_{1}\right)$. In Scenario I, the firm is considering introducing the FP but is not currently in the market for the other SPs. In scenario II, we create two sub-scenarios: (II.1) the supplier of the FP is the supplier of SP 1; (II.2) the supplier of the FP is the supplier of SP 2. In Scenario III, the firm produces both SPs as well as the FP. For each scenario, we conduct ten comparisons as mentioned above and evaluate the influence on the optimal launch time of the FP and the total profit.

The parameter values of the base case are: $m_{1}=100, m_{2}=200, m_{3}=200, d_{1}=1.5, d_{2}=$ $2.5, d_{3}=4, \phi_{1}=\phi_{2}=0.0049, \psi_{1}=\psi_{2}=0.644, \phi_{3}=\kappa \phi_{1}, \psi_{3}=\kappa \psi_{1}, t_{1}=2, t_{2}=4, T_{3}=20, t_{m}=$ $4, R_{1}=0.5, R_{2}=0.3, w=0.7, \kappa=1, \alpha=10, v_{0}=280$. The base case has the following properties: (1) the FP has a unit profit which equals the sum of the two SPs' unit profits, (2) the two SPs and the FP have the same diffusion parameters, (3) the two SPs are still 'young' and the fusion technology will be mature soon (in four periods). We consider multifunction office machines as one example. The printer is represented by SP 2 and the scanner is represented by SP 1 . Since the fusion product normally integrates closely related functions and both the printer and the scanner are PC peripherals, setting the same innovation and imitation parameters for two SPs is reasonable. The innovation and imitation parameter values used in our examples are based on the diffusion parameters from Bass, Krishnan and Jain (1994). The variable $\kappa$ indicates the diffusion speed multiplier of the FP with respect to the diffusion speed of the SPs 1 and 2 . When $\kappa$ is greater than one, the FP has a faster diffusion speed than the SPs. In the base case, we assume that the diffusion speed of the FP is the same as the diffusion speed of the SPs.

Based on the scenarios we describe above, the fusion product manufacturer can be one of four different roles (I) a total new entrant, (II.1) a manufacturer of SP 1, (II.2) a manufacturer of SP 2 , and (III) a manufacturer of two SPs. Table 2 lists the optimal solutions from all cases and all

four scenarios conducted in this paper. The optimal solutions in Table 2 are found through a full range search of the profit curve on various launch times. In section 4.1 we contrast the impact of different launch times on the diffusion processes. In section 4.2, we compare the results among four scenarios. The impacts of parameter changes on are discussed in sections 4.3 to 4.6 and summarized in section 4.7. Finally, a discussion of the sensitivity of the profit to changes in these parameters is included in section 4.8 .

Insert Table 2 here

\subsection{Market Penetration}

The launch of the fusion product often changes the dynamics of related single-function products. In this subsection, we exemplify the cumulative sales of the SPs and the FP for the base case example, which are shown in Figure 2. The original cumulative sales of SPs without the competition of the FP 
are represented by thin solid lines, where the SP 1's (2's) diffusion has a lower (higher) cumulative sales curve with $t_{1}=2$ and $m_{1}=100\left(t_{2}=4\right.$ and $\left.m_{2}=200\right)$. The diffusion of the FP is represented by a thick solid line and the long dashed line approaching 200 represents the potential cumulative sales of the FP solely from $m_{3}$. The short dashed lines represent the cumulative sales of SPs 1 and 2 after the launch of the FP.

Insert Figure 2 here

Figures 2.1 and 2.2 are intended to illustrate the impact of alternate fusion product launch times on the diffusion for all products. Figure 2.1 shows the market penetration if the FP is launched at $\tau=1$. As the FP is launched, the cumulative sales for SPs 1 and 2 increase by smaller rates as some potential adopters switch to buy the FP. As a result of the launch of the fusion product, the cumulative sales of SPs 1 and 2 drops from 100 to 52.1 and from 200 to 149.4 at the end of the planning horizon. Conversely, the cumulative sales of FP 3 increases from 200 to 241.4. The total volume of sales loss from two SPs is 98.5 units but the switch only increases the sales volume of FP by 41.1 units. The difference is due to the overlap of consumers. Specifically, consumers who were in the marketplace for both individual SPs will now only buy a single FP.

If the FP is launched later, such as in Figure 2.2 at $\tau=3$, then the impact of the FP on the SPs is moderated. The cumulative sales of SPs remain intact before the FP is launched, but the total sales of the FP are lower. The cumulative sales of SPs 1 and 2 are 57.8 and 164.7, which are higher than those in Figure 2.1, and the cumulative sales of the FP is 231.6.

Next, we investigate the factors which influence the firm's optimal launch time decision for the fusion product.

\subsection{Role of the Fusion Product Supplier}

First, we investigate the role of the FP manufacturer in four different scenarios. The results in Table 2 and ten sub-figures in Figure 3 show that the more substitution that the FP has with its current SP(s), the later the firm should introduce the FP. The cannibalization effect is highest in Scenario III, then Scenario II.2, then Scenario II.1, then Scenario I, where there is no cannibalization whatsoever.

The results are not surprising because a new entrant has nothing to lose. A firm who offers both SPs and the FP must be concerned with (1) the increased sales of the FP which comes with some decreased sales of SPs, and (2) some future adopters choose to "buy one FP instead of two SPs," which is reflected by parameter $w$. We should note that the cannibalization is captured by many factors, including the relative magnitudes of total future adopters, unit profits, the switching rate, and the degree of the overlap. Different scenarios also reflect different cannibalization effects depending on the firm's mix of current product offerings. 
Insert Figure 3 here

\subsection{Synergy of the FP: Faster Diffusion Speed, Higher Margin, and Market Expansion}

In section 1, we mention that the synergy of the FP may include space saving, lower total purchasing cost, and function synchronization. There are several ways that the synergy of the FP is reflected in the model. First, when two functions have greater synergy, the diffusion of the FP may be faster. Second, when the FP has greater synergy to integrate two functions together, it normally has a higher unit profit. Third, the FP might have a larger number of future adopters when it has greater synergy.

Norton and Bass (1987) use the same innovation and imitation parameters to fit the diffusion data of different generations of DRAM. However, van den Bulte and Lilien (1997) point out that this model does not account for the decline of real prices, improving product performance, and increasing distribution penetration. These factors increase the total adopters and result in a downward pressure on the estimates (i.e., $\psi$ and $\phi$ ). Van den Bulte (2000) investigates the changes of the diffusion speed of 31 electrical durables in the United States from 1923 to 1996. He defines diffusion speed as the time to reach a certain market penetration level and measures it by the slope coefficient of the logistic diffusion model. The variance of diffusion speed is primarily explained by the purchasing power, the demographics, and the maturity of the products. Excluding the variance, this study finds that the diffusion speed is increasing with statistical significance.

Based on the above research, we use a multiplier $\kappa$ to represent whether the FP has an equal or larger diffusion speed than its component SPs. In the base case we set the innovation and imitation parameters of the FP at the same level as the SPs $(\kappa=1)$. In cases 1 to 3 , we set $\kappa$ at $1.1,1.2$, and 1.3, respectively. Table 2 shows that, in all scenarios, the diffusion speed has no significant impact on the optimal launch time and the total profit when other parameters remain unchanged. Even though the diffusion speed is important to forecast when the demand of FP will grow faster, our results shows the optimal launch times are very similar despite the different diffusion speed.

If the synergy of the two functions is reflected on the FP's unit profit, then the FP's optimal launch time is strongly influenced. Cases 4, 5, and 6 are comparable situations to the base case with alternate values for the FP's unit profit. The results in Figure 3.2 show that when the FP is less profitable, the launch time should be postponed to more closely match the time of technology maturity. The development cost structure in our model makes our optimal launch time different from Wilson and Norton's (1989) "now or never" and from Mahajan and Muller's (1996) "now or at-peak" conclusions. When there is a higher unit profit for the FP, the launch time should be earlier in order to capture more demand from the SPs. It should be noted that the FP's optimal 
launch time should be no later than the technology cost maturity $\left(t_{m}\right)$, which occurs when the FP has a relatively high unit profit compared with the two SPs together. If the FP is less profitable, it is possible that the optimal launch time can be postponed until after $t_{m}$ under a certain circumstance. For example, when the FP's profit is only slightly larger than SP 2, the launch of the FP is delayed.

Kurawarwala and Matsuo (1998) find the innovative and imitative parameters are easy to assess, but the magnitude of the total adopters has high uncertainty. Cases 7, 8, 9 and the base case show that the FP's optimal launch time is insensitive to various market sizes of the FP (Figure 3.3) when the cannibalization is strong. The results mean that if the higher synergy of the FP is represented by more future adopters while other conditions remain the same, then the FP's optimal launch time is not much different from the case with a lower synergy FP for firms with existing products. For the firms in scenarios I and II.1, the FP's market size has stronger influence on the optimal launch time.

\subsection{Maturity of the Fusion Technology and the Development Cost}

When the initial fusion technology which combines two functions is available, the technology might not be mature immediately. If the fusion technology has a longer development time, speeding up the launch of the FP normally requires a higher level of product development investment. The product development cost might have different increasing rates in different circumstances. For some FPs like office machines, the convex development cost might have a lower rate of increase. For other FPs, such as the PDA phone, the convex development cost may increase at a steeper rate since the integration is more challenging. In this section, we analyze the optimal launch time of the FP under various times to technology maturity and various development cost curves.

Cases 10,11, 12 and the base case compare different slopes of the development cost curves, which is represented by $\alpha$. Figure 3.4 shows that a development cost curve with a steep slope, which has a larger $\alpha$, leads to a later optimal launch time of the FP. Furthermore, the impact on the optimal time to market is sizable. As time-to-market becomes more costly, the firm should wait until the fusion technology is more mature.

Cases 13, 14, 15 and the base case compare various values for the technology cost maturity. We find that the FP's optimal launch time should be early when the fusion technology's maturity is early. When a fusion technology will be mature immediately, it implies the integration of the separate functions is not difficult. The technology break-through of chip design in recent years shows the sign of a mature fusion technology, therefore many fusion products are available soon after a new function (e.g., MP3 player, digital camera) is invented and offered in the market. If the fusion technology's maturity is late, the convex cost curve hinders the firm from an early launch of the FP. 


\subsection{Time Related Factors}

So far, our numerical examples are all based on two SPs that are in their early stages of life cycles: SPs 1 and 2 were just launched for 2 and 4 periods, respectively. As technology advances at an increasing pace, the fusion technology is often available soon after a new function is invented. However, the gap between two SPs' launch times might be several years or more than a decade. For example, the time gap between the first cell phone and first PDA launched in the U.S. market is 17 years.

In this section, we investigate the impact of the ages of the SPs on the timing of the introduction of the FP as shown in Figure 3.6. Specifically, we investigate the impact of various values for $t_{2}$. Cases 16, 17, and 18 and the base case show that the firm in scenarios I or II.1 should pursue an early launch of the FP when the SP 2 is currently 'older'. Interestingly, the firm in these two scenarios does not have control of the SP 2, but rather has to respond strategically to changes in the age of this product. In contrast, the fusion product launch time for the firm in scenarios II.2 and III is non-linear in the age of SP 2. A likely cause of this result is the steep flexion point of the sales curve for SP 2. Nonetheless, the relative magnitude of these effects is fairly constant regardless of the age of SP 2. When the cannibalization is strong in these scenarios (i.e. when the firm launching the FP will cannibalize sales of its own SP 2), then the age of the SP 2 is not as influential on the FP launch time.

Cases 19, 20, 21 and the base case compare the optimal launch time of the FP for different values of the length of the planning horizon. Similar to Wilson and Norton (1989), we find that the planning horizon is a decisive factor for the optimal launch time when it is short. If the planning horizon is long enough, then it does not influence the optimal launch time much. When $T_{3}$ is only ten periods, the optimal launch time for the FP is 'now'; however, when $T_{3}$ is 25 periods, the optimal launch time is similar to when $T_{3}$ is 20 periods. We find that a short planning horizon will make the diffusion speed (i.e., $\kappa>1$ ) of the FP become influential. If the planning horizon is short, then a faster diffusion speed will shorten the diffusion process; as a result, it induces the firm to launch the FP early.

\subsection{Market Size, Substitution and Overlap}

Figure 3.8 shows the changes of $m_{1}$ to investigate the impact of SP's relative size of total future adopters. Changing $m_{1}$ from 50 to 200, the total future adopters ratio between SPs 1 and 2 varies from 1:4 to 1:1. For all scenarios, smaller $m_{1}$ leads to a later optimal launch time of the FP. However, the impact is moderate, especially for the firm in scenario II.1. The firm in scenario II.1 currently offers SP 1 . Launching the FP will primarily change its revenue source from SP 1 to the FP, but the total revenue barely increases. Hence, the firm in scenario II.1 has less incentive to 
launch the FP, which induces a late launch of the FP. Note that when two SPs have a similar size of future adopters, (i.e. $m_{1}=m_{2}$ ), the gap of the optimal launch times between scenarios II.1 and II.2 is also smaller, which means the two scenarios have more similar cannibalization concerns.

To address the substitution effects, we investigate the impact of different degrees of switching rate $R_{2}$. A larger value of $R_{2}$ means that a higher proportion of future adopters may switch to the FP. The higher the $R_{2}$ is, the higher proportion in demand of SP 2 will be substituted for the FP. Cases 22, 23, 24 and the base case compare the impact of different degrees of $R_{2}$. The results in Figure 3.9 shows that when the FP's substitution rate is higher, the firm should launch the FP early. Note that the impact of $R_{1}$ is symmetric to $R_{2}$.

To avoid repeatedly counting the switches from two SPs, we use a parameter $w(w \leq 1)$ to adjust the total switches from two SPs. When $w$ is larger (smaller), there are fewer (more) overlapped future adopters who would "buy one FP instead of two SPs." A lower bound of $w$ should be the minimum of switches from SP 1 and SP 2. The last comparison is to investigate the overlap degree of the future adopters from two SP markets.

Finally we change the value of $w$ to $0.6,0.8$, and 0.9 in cases 28,29 , and 30 , respectively, in contrast to the base case with $w=0.7$. Figure 3.10 shows that when there is more overlap between the two SPs' future adopters, the firm should introduce the FP later because the buyers from the SPs will eventually purchase just one FP rather than two SPs. This result implies that when the firm intends to launch the FP to "hit two birds with one stone," they need to carefully estimate how many future buyers for the markets of the two SPs will eventually buy just one FP.

\subsection{Summary of the Optimal Launch Time}

Wilson and Norton (1989) find that the substitution effects, the diffusion between two generations of similar products, the planning horizon, and the relative profitability are major factors influencing the optimal launch time of the new product. Our numerical examples support their conclusion. Mahajan and Muller (1996) demonstrate that the relative market sizes between generations are also influential to the introduction time. In our product diffusion models, the numerical analyses show that the market size of the FP matters more for the firm with less cannibalization concern. Besides, there are additional factors impacting the launch decision of the fusion product: (1) the shape of the development cost curve, (2) the technology cost maturity, (3) the age of the earlier launch SP (4) the overlap degree from two SP markets, and the most important (5) the competitive role of the fusion product supplier. The influence of the FP's market size and the diffusion speed of the FP are not notable for these numerical studies.

In terms of the demand model structure, our model is similar to Mahajan and Muller (1996). However, while the firm also considers the convex development cost, the optimal launch time is

often between 'now' and the technology cost maturity when the FP is relatively lucrative. In some 
extreme cases (i.e., short planning horizon or an FP with very low profit margin), the optimal launch time is either "now" or "at peak (of the SP)."

Here we summarize the insights from the numerical examples.

1. The greater the cannibalization concern of the firm, the later the FP should be launched.

2. The optimal launch time is moved later as the following factors increase: the steepness of the development cost curve, the technology cost maturity, the planning horizon, and the overlap degree from two SP markets.

3. The optimal launch time should be set earlier as the following factors increase: the FP's profit margins and the switching rate from two SPs to a single FP.

4. If the FP is profitable enough to offset the lost sales of SPs, the FP's optimal launch time will be between 'now' and the technology cost maturity. When the FP is more profitable or the early-launch is less costly, the firm should introduce the FP earlier.

5. When the planning horizon is relatively long, the market size and the diffusion speed of the FP do not have influential impact on the optimal launch time of the FP.

6. The ages of the SPs show strong non-linearity on the optimal launch time when the firm has increased cannibalization concerns. The optimal launch time is convex decreasing then increasing as the ages of the SPs increases from young to mature.

\subsection{Impact on the Total Profit}

The analysis above focuses on the optimal launch time of the FP. Now we evaluate the financial performance of the launch timing decision. Figure 4 demonstrates the impact of parameter changes on the profit. For each sub-figure and each scenario, the profit from the base case is set as the reference. The total profits of other cases due to some parameter changes are shown as ratios relative to the profit of the base case. In most sub-figures, the ratios are shown in a moderate range of $[0.9,1.1]$. In Figures 4.2, 4.3, 4.8 and 4.9, the ratios are shown in a large range which can properly demonstrate their total variation.

Insert Figure 4 here

We find that the diffusion speed is not influential to the total profit. We can easily tell that the diffusion speed (Figure 4.1) given a relatively long planning horizon $\left(T_{3}=20\right)$ does not impact the profit for all scenarios. The FP's market size is not influential to the optimal launch time in scenario III, however, the market size (like the unit profit margin) has a large impact on its total profit in Figure 4.3. 
From Figure 4, we can obtain the following insights.

1. If the synergy of the FP is reflected by a higher profit margin or a larger market size, then the synergy has a strong impact on the total profit in all scenarios.

2. The diffusion speed has no influential impact on the total profit when the planning horizon is long enough.

3. A short planning horizon and the switching rates of two SPs to FP have moderate impact on the total profit, especially when the firm offers three products and has a greater cannibalization concern.

4. When the firm has the maximum cannibalization concern as in scenario III, the impact on the total profit from the factors (except the market sizes and profit margins) is more moderated than in other scenarios.

5. When the firm has a minimum cannibalization concern as in scenario I, the optimal launch decision has a greater impact on the total profit than a firm which has higher cannibalization. This result implies that a new entrant should be more careful when it decides the launch time of the FP.

\section{Conclusion and Future Research}

As fusion products become more prevalent in electronic markets, product diffusion becomes more complex as compared to the diffusion process within one product category. This is the first product diffusion model that investigates the dynamics of two SPs and one FP simultaneously, as a stylized product diffusion model is constructed that is as parsimonious and complete as possible. Incorporating both development costs and accounting for consumers that may replace two SPs with a single FP device, the model offers managerial guidance concerning the optimal launch time of the FP. Due to the complexity of the model, no explicit closed form analytical solutions are derived. However, we conduct several sets of numerical comparisons showing the impact of parameter changes on the FP's optimal launch time and total profit.

We find that the competitive role of the FP's manufacturer is influential to the launch decision of the FP. When the supplier of the FP is also the supplier of the $\mathrm{SP}(\mathrm{s})$, then the higher cannibalization results in a later launch time of the FP. Moreover, the timing of the entry into the FP market is particularly crucial to the profitability of a new entrant. This finding suggests that the firms involved should evaluate who owns the fusion technology and carefully assess the profitability, cost and diffusion factors associated with both the FP and the SPs. 
Because we incorporate many realistic functions for the development cost and the maturity time of the fusion technology, the optimal launch time policy is different from the conclusions of Wilson and Norton's (1989) "now or never" and from Mahajan and Muller's (1996) "now or at-peak" When the FP is relatively profitable (normally it is), the optimal launch time of the FP is between 'now' and the maturity of the fusion technology. In general, the higher the profit margin associated with the FP, the earlier the FP should be launched.

Our numerical examples demonstrate the impact of parameter changes on the optimal launch time and the total profit. A summary of the highlights of these examples follows:

1. When the FP has a higher profit margin, the firm should launch the FP earlier. The total profit of the firm is also very sensitive to changes in the FP profit margin.

2. The diffusion speed of the FP is significant only when the planning horizon is short. Otherwise, the diffusion speed is not influential to the optimal launch time or the total profit.

3. An increase in the market size for the FP influences profit, but is not a significant driver of the launch time.

4. A flatter development cost curve and early maturity of the fusion technology imply an earlier launch. For our numerical examples, these development cost parameters did not have a large influence on total profit.

5. A higher consumer switching rate from the SP to the FP also leads to an earlier launch. However, if more consumers are consolidating from two SPs to a single FP device, then the firm should launch the FP later.

There are several directions exploring the diffusion of FPs that warrant further research. First, an empirical fitting of the diffusion parameters of the FP is strongly needed in many product markets, especially in personal mobile device, PC, and office machine markets. As we mentioned above, a well-categorized study of the statistics for the sales of related products is a key stepping stone for this research. Without reasonable categorization, any FP diffusion fitting would be biased. This is especially challenging because many FPs integrate more than four or five functions in one device.

For example, Chen et al. (2008b) show the complexity of a product portfolio with multiple correlated functions. Several questions remain which should be addressed via empirical research in the future: Whether the diffusion speed of the FP is significantly different from its component SPs? Do two products have a similar diffusion pattern when they have higher similarity and substitution effects? Does a new FP capture most of its demand from other SPs or FPs? Or does a new 
FP generate a significant amount of new potential adopters? An empirical study answering these questions has the potential to make a solid contribution to the FP literature.

Second, many SPs still thrive after the launch of the FP, but others become lack-luster. Firms may try to rejuvenate the market for the SPs by adding new functions, improving the quality and identifying new applications for the product. For example, after the launch of the inkjet office machine FP, an additional market for the high end inkjet SP helped to fuel future growth: digital photo printing. A research study that further characterizes the properties of a thriving SP would provide constructive guidelines to these industries.

Third, a research survey from the consumer's point of view will help to answer the following questions. How do consumers perceive the FP and which component SP is more similar (or substitutable) to the FP? What component SPs will be replaced if the consumer buys one FP? Why do some FPs replace component SPs very quickly, but others do not? Why does the demand of one component SP shrink very quickly after the launch of the FP, but another component SP is resistant to the FP? We recommend that conjoint analysis may be an appropriate tool to utilize for this type of FP and SP market research.

Finally, an explicit treatment of competition on the FP decision making is warranted. From manufacturers in all SPs, which firm has a competitive advantage in offering a fusion product? The transformation of electronics product market provides many good cases which offers insights in product design and offering strategy for the manufacturers. 


\section{References}

ABI Research. 2005. Mobile Phone Imaging. http://www. abiresearch.com/products/market_ research/Mobile_Phone_Imaging. August 292005.

Anonymous. 1997. A Fax Machine or More? Consumer Reports. 62(9) 32.

Avery, S. 2003. Purchasing strategy now focuses on value of MFPs. Purchasing. 132(12) 53.

Avery, S. 2004. FP Demand Strengthens; HP Enters Crowded Market. Purchasing. 133(1) 48.

Bass, F. M. 1969. A New-Product Growth Model for Consumer Durables. Management Science. 15(5), 215-227.

Bass, F. M., T. V. Krishnan, D. C. Jain. 1994. Why the Bass Model fits without Decision Variables.Marketing Science. 13(3), 203-223.

Bayus, B. L. 1997. Speed-to-Market and New product performance Trade-offs. Journal of Product Innovation management. 14(6) 485-497.

Bemmaor, A. C., J. Lee. 2002. The Impact of Heterogeneity and Ill-Conditioning on Diffusion Model Parameter Estimates. Marketing Science. 21(2), 209-220

Breeden, J. II, C. A. de Soto. 2000 OFFICE GEAR; Multi-function Devices Demand Fewer Compromises. The Washington Post Oct 26, 2000, E11.

Cellular-news.com. 2006. PDA Market Shrinks As Mobiles Take Customers. http://www.cellular-news . com/story/17163.php, April 28, 2006.

Cellular-news.com. 2006. Gartner Says PDA Shipments Grew 32 Percent in Q3. http://www. cellular-news.com/story/20464.php, November 172006.

Cellular-news.com. 2006. Worldwide PDA and Smartphone Shipments Market Grew 57 Percent. http://www. cellular-news.com/story/19802.php, October 112006.

Chen, Y., A. J. Vakharia, A. Alptekinoğlu. 2008. Product Portfolio Strategies: The Case of Multi-Function Products. Production \& Operations Management. 17(6) 587-598.

Chen, Y., A. J. Vakharia, J. Carrillo, P. Sin. 2009. Fusion Product Planning: A Market Offering Perspective. Working paper. University of Florida, Gainesville, FL.

Cullen, S. 1999. To FP or not to FP. Office Systems. 16(9) 36.

Ferguson, S. 2006. Study: Smart Phone, PDA Shipment Up 57 Percent. http://www.eweek.com/ article2/0, 1895, 2027671,00.asp, October 102006.

Fisher, J. C., R. H. Pry. 1971. A Simple Substitution Model of Technology Change. Technological Forecasting 8 Social Change. 3(March), 75-88. 
Gartner. 2003. Gartner Says Latin American Printer Shipments Declined 5 Percent in the First Quarter of 2003 All-in-one Printer Shipments Increased 176 Percent, http://www.gartner. com/press_releases/pr14may2003d.html, May 14, 2003.

Gilroy, A. 2004. Smartphone Sales On Pace To Overtake PDAs. TWICE. Sep 20, 2004. 19(19) 8.

Givon M., V. Mahajan, E. Muller. 1995. Software Piracy: Estimation of Lost Sales and the Impact on Software Diffusion. Journal of Marketing. 59(1) 29-37.

Gupta, A. K., K. Brockhoff, U. Weisenfeld. 1992. Making Trade-Offs in the New Product Development Process: A German/US Comparison. Journal of Product Innovation Management. 9(1) $11-18$.

Ho, T. H., S. Savin, C. Terwiesch. 2002. Managing Demand and Sales Dynamics in New Product Diffusion Under Supply Constraint. Management Science. 48(2), 187-206.

Harbaugh, L. 1998. Find the Right Balance When Combining Functions. Information Week. Jun 29, 1998, 120 .

Jain, D., V. Mahajan E. Muller. 1991. Innovation Diffusion in the Presence of Supply Restriction. Marketing Science. 10(1), 83-90.

Kim, N., D. R. Chang, A. D. Shocker. 2000. Modeling Intercategory and Generational Dynamics for a Growing Information Technology Industry. Management Science. 46(4) 496-512.

Kurawarwala, A. A., H. Matsuo. 1998. Product Growth Models of Medium-Term Forecasting of Short-Life-Cycle Products. Technological Forecasting 8 Social Change. 573), 169-196.

Kim, N., V. Mahajan, R. K. Srivastava. 1995. Determining the Going Value of a Business in an Emerging Information Technology Industry: The Case for Cellular Communications Industry. Technological Forecasting \& Social Change, 49(3), 257-279.

Liberman, M. B., D. B. Montgomery. 1988. First-Mover Advantages. Strategic Management Journal 9(2), 41-58.

Magid, L. J. 1998. The Cutting Edge / Personal Technology; All-in-One Devices Improve. Los Angeles Times Apr 20, 1998, 5

Mahajan, V., E. Muller, F. M. Bass. 1990. New Product Diffusion Models in marketing: A Review and Directions for Research. Journal of Marketing. 54(1), 1-26.

Mahajan, V., E. Muller. 1996. Timing, Diffusion, and Substitution of Successive Generations of Technological Innovations: The IBM Mainframe Case. Technological Forecasting 85 Social Change. 51 109-132.

Mahajan, V., E. Muller, Y. Wind, ed. 2000. New Product Diffusion Models. Kluwer Academic Publishers, Boston, MA. 
Mahajan, V., S. Sharma, R. A. Kerin. 1988. Assessing Market Opportunities and Saturation Potential for Multi-Store, Multi-Market Retailers. Journal of Retailing. 64(3), 315-333.

Mahajan V., S. Sharma, R. D. Buzzell. 1993. Assessing the Impact of Competitive Entry on Market Expansion and Incumbent Sales. Journal of Marketing. 57(3) 39-52.

Meyers, J. 2004. Game Theory, Teen-Style. American Demographics 24(4) 10.

Min S., M. U. Kalwani, W. T. Robinson. 2006. Market Pioneer and Early Follower Survival Risks: A Contingency Analysis of Really New Versus Incrementally New Product-Markets. Journal of Marketing 70(1) 15-33.

Murmann, P. A. 1994. Expected Development Time Reductions in the German Mechanical Engineering Industry. Journal of Product Innovation Management 11(3) 236-252.

Norton, J. A., F. M. Bass. 1987. A Diffusion Theory Model of Adoption and Substitution for Successive Generations of High-Technology Products. Management Science. 33(9) 1069-1086.

Pae, J. H., D. R. Lehmann. 2003. Multigeneration innovation diffusion: The impact of intergeneration time. Academy of Marketing Science Journal. 31(1), 36-47.

Palminfocenter.com. 2006. IDC: Handheld Sales Continue Decline. http://www.palminfocenter.com/ news/8866/idc-handheld-sales-continue-decline, August 32006.

Putsis, W. P., Jr. 1989. Product Diffusion, Product Differentiation and the Timing of New Product Introduction: The Television and VCR Markets 1964-1985. Managerial and Decision Economics. 10(1), 37-50.

Smith, P.G., D. G. Reinertsen. 1991. Developing Products In Half The Time. $4^{\text {th }}$ ed., Van Nostrand Reinhold, New York.

Roger, E. M. 2003. Diffusion of Innovations. $5^{\text {th }}$ ed., Free Press, New York, NY.

Rust, R. T., D. V. Thompson, R. W. Hamilton. 2006. Defeating Feature Fatigue. Harvard Business Review. 84(2) 98-107.

Rysavy, P. 2004. Making The Smart Choice. Network Computing. 15(7) 53.

Schmidt, G. M., E. L. Porteus. 2000. Sustaining Technology Leadership Can Require Both Cost Competence and Innovative Competence. Manufacturing $\&$ Service Operations Management, 2(1) $1-18$.

Schonfeld, E. 2004. BlackBerry Season Riding a Huge Comeback, Research in Motion Finds Itself Surrounded by Rivals, All Gunning for A Share of the Booming Wireless Market It Created.Business 2.0 5(9) 132-140.

Sohn, Y. S., B. J. Ahn. 2003. Multigeneration Diffusion Model for Economic Assessment of New 
Technology. Technological Forecasting \&3 Social Change. 70(3) 251-264.

Talukdar, D., K. Sudhir, A. Ainslie. 2002. Investigating New Product Diffusion across Products and Countries. Marketing Science. 21(1), 97-114.

Thompson, D. V., R. W. Hamilton, R. T. Rust. 2005. Feature Fatigue: When Product Capabilities Become Too Much of a Good Thing. Journal of Marketing Research 42(4), 431-42.

Van den Bulte, C., G. L. Lilien. 1997 Bias and Systematic Change in the Parameter Estimates of Macro-Level Diffusion Models. Marketing Science. 16(4), 338-353

Van den Bulte, C. 2000. New Product Diffusion Acceleration: Measurement and Analysis. Marketing Science. 19(4), 366-380.

Van den Bulte, C. 2004. Multigeneration Innovation Diffusion and Intergeneration Time: A Cautionary Note. Academy of Marketing Science. Journal.32(3), 357-360.

Wilson, L. O., J. A. Norton. 1989. Optimal Entry Timing for A Product Line Extension. Marketing Science 8(1) 1-17. 
Table 1. Notation and Acronyms.

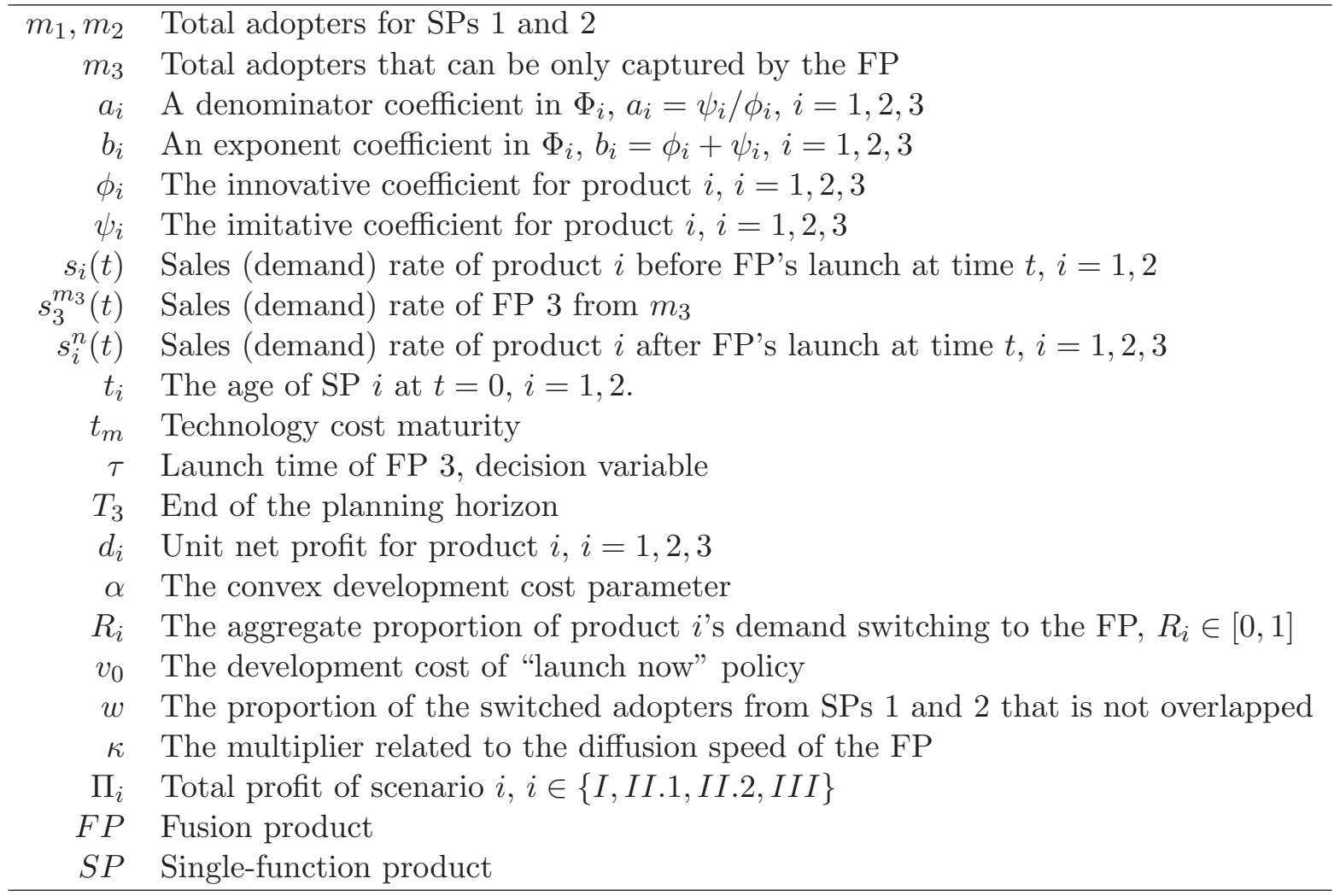


Table 2. Optimal launch time of FP and the profit under four scenarios and 31 cases.

\begin{tabular}{|c|c|c|c|c|c|c|c|c|c|}
\hline Case & Difference & $\tau_{I}^{*}$ & $\Pi_{I}^{\tau^{*}}$ & $\tau_{I I .1}^{*}$ & $\Pi_{I I .1}^{\tau^{*}}$ & $\tau_{I I .2}^{*}$ & $\Pi_{I I .2}^{\tau^{*}}$ & $\tau_{I I I}^{*}$ & $\Pi_{I I I}^{\tau^{*}}$ \\
\hline 0 & Base case & 1.5167 & 832.76 & 1.6857 & 909.74 & 2.6024 & 1180.33 & 3.0166 & 1262.86 \\
\hline 1 & $\kappa=1.1$ & 1.5306 & 833.22 & 1.7027 & 910.25 & 2.6375 & 1181.24 & 3.0706 & 1264.03 \\
\hline 2 & $\kappa=1.2$ & 1.5353 & 833.36 & 1.7085 & 910.41 & 2.6504 & 1181.53 & 3.0915 & 1264.44 \\
\hline 3 & $\kappa=1.3$ & 1.5369 & 833.40 & 1.7104 & 910.46 & 2.6551 & 1181.63 & 3.0993 & 1264.57 \\
\hline 4 & $d_{3}=3$ & 1.8617 & 567.94 & 2.1009 & 646.31 & 3.2495 & 925.74 & 3.8687 & 1013.65 \\
\hline 5 & $d_{3}=5$ & 1.2435 & 1099.51 & 1.3730 & 1175.59 & 2.1058 & 1440.16 & 2.3808 & 1519.67 \\
\hline 6 & $d_{3}=6$ & 1.0182 & 1367.62 & 1.1225 & 1443.08 & 1.7240 & 1703.42 & 1.9190 & 1781.17 \\
\hline 7 & $m_{3}=150$ & 1.5219 & 632.93 & 1.6921 & 709.92 & 2.6159 & 980.66 & 3.0375 & 1063.30 \\
\hline 8 & $m_{3}=250$ & 1.3060 & 866.50 & 1.4449 & 942.77 & 2.5607 & 1297.56 & 2.9640 & 1397.80 \\
\hline 9 & $m_{3}=300$ & 1.1223 & 901.07 & 1.2393 & 976.81 & 2.5194 & 1414.81 & 2.9121 & 1496.78 \\
\hline 10 & $\alpha=5$ & 0.6603 & 853.37 & 0.8157 & 928.09 & 1.9407 & 1187.49 & 2.4337 & 1266.70 \\
\hline 11 & $\alpha=15$ & 1.9995 & 820.35 & 2.1738 & 899.17 & 2.9313 & 1176.60 & 3.2809 & 1261.09 \\
\hline 12 & $\alpha=20$ & 2.3239 & 811.97 & 2.4972 & 892.30 & 3.1328 & 1174.29 & 3.4329 & 1260.07 \\
\hline 13 & $t_{m}=2$ & 0.4781 & 872.58 & 0.5802 & 946.90 & 1.2698 & 1201.29 & 1.4914 & 1277.57 \\
\hline 14 & $t_{m}=3$ & 1.0151 & 855.08 & 1.1464 & 930.57 & 1.9604 & 1192.48 & 2.2696 & 1271.41 \\
\hline 15 & $t_{m}=5$ & 1.9993 & 805.35 & 2.2208 & 884.27 & 3.2127 & 1164.43 & 3.7502 & 1251.70 \\
\hline 16 & $t_{2}=3$ & 1.8422 & 848.99 & 2.0447 & 927.21 & 2.6424 & 1203.86 & 3.0541 & 1286.63 \\
\hline 17 & $t_{2}=5$ & 1.1855 & 812.03 & 1.3358 & 887.98 & 2.5867 & 1141.84 & 3.0165 & 1224.31 \\
\hline 18 & $t_{2}=6$ & 0.9114 & 787.24 & 1.0676 & 862.50 & 2.6085 & 1084.31 & 3.0580 & 1166.98 \\
\hline 19 & $T_{3}=10$ & 0 & 810.84 & 0 & 884.37 & 0 & 1130.87 & 0 & 1204.39 \\
\hline 20 & $T_{3}=15$ & 1.1249 & 818.52 & 1.2334 & 894.10 & 1.8191 & 1155.81 & 1.9999 & 1233.75 \\
\hline 21 & $T_{3}=25$ & 1.5368 & 833.41 & 1.7104 & 910.47 & 2.6554 & 1181.65 & 3.1002 & 1264.60 \\
\hline 22 & $m_{1}=50$ & 1.6735 & 767.36 & 1.7698 & 806.06 & 2.9839 & 1120.04 & 3.2542 & 1162.34 \\
\hline 23 & $m_{1}=150$ & 1.3819 & 898.60 & 1.6087 & 1013.55 & 2.3167 & 1242.36 & 2.8187 & 1364.01 \\
\hline 24 & $m_{1}=200$ & 1.2632 & 964.78 & 1.5375 & 1117.46 & 2.0886 & 1305.55 & 2.6498 & 1465.66 \\
\hline 25 & $R_{2}=0.2$ & 1.8519 & 789.47 & 2.0818 & 867.79 & 2.6589 & 1176.46 & 3.0874 & 1259.38 \\
\hline 26 & $R_{3}=0.4$ & 1.2421 & 877.93 & 1.3729 & 954.02 & 2.5469 & 1184.25 & 2.9467 & 1266.40 \\
\hline 27 & $R_{4}=0.5$ & 1.0121 & 924.50 & 1.1176 & 999.95 & 2.4923 & 1188.22 & 2.8778 & 1270.01 \\
\hline 28 & $w=0.6$ & 1.6992 & 795.33 & 1.9019 & 873.01 & 2.9427 & 1148.11 & 3.4630 & 1233.25 \\
\hline 29 & $w=0.8$ & 1.3562 & 870.79 & 1.5007 & 947.23 & 2.3081 & 1214.17 & 2.6361 & 1294.80 \\
\hline 30 & $w=0.9$ & 1.2132 & 909.32 & 1.3390 & 985.31 & 2.0546 & 1249.28 & 2.3184 & 1328.53 \\
\hline
\end{tabular}

* The parameter values of base case are: $m_{1}=100, m_{2}=200, m_{3}=200, d_{1}=1.5, d_{2}=2.5, d_{3}=4$, $\phi_{1}=\phi_{2}=0.0049, \psi_{1}=\psi_{2}=0.644, \phi_{3}=\kappa \phi_{1}, \psi_{3}=\kappa \psi_{1}, \kappa=1, t_{1}=2, t_{2}=4, T_{3}=20$, 


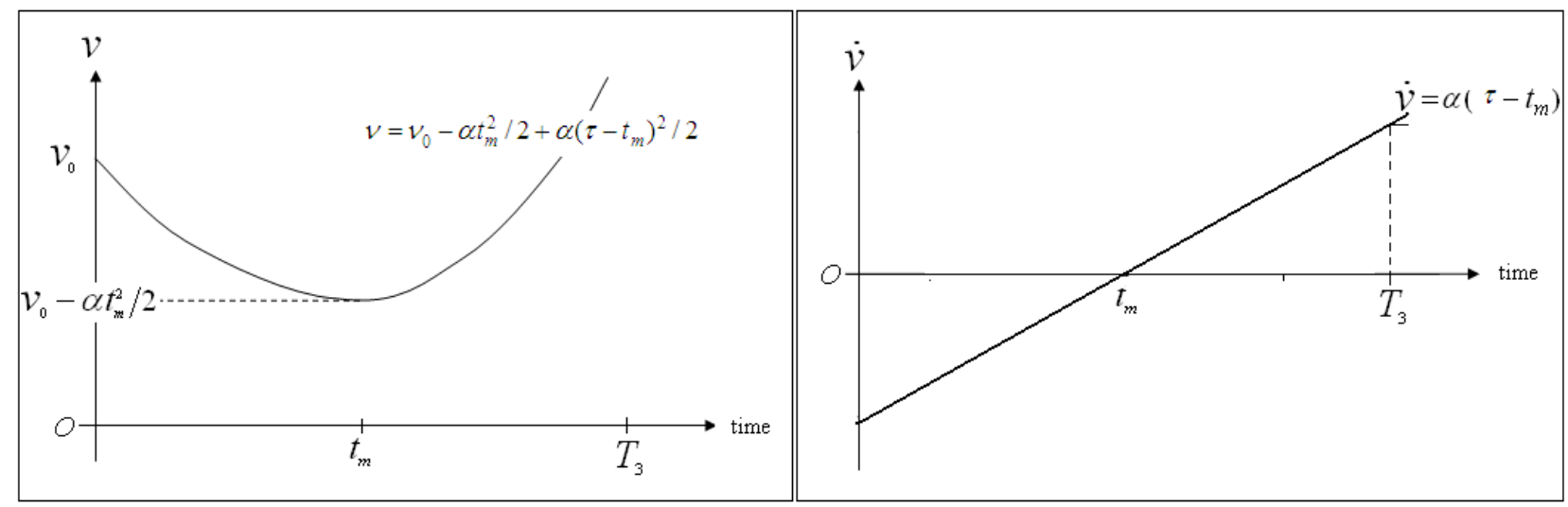

Figure 1. Development Cost at Different Launch time of FP.

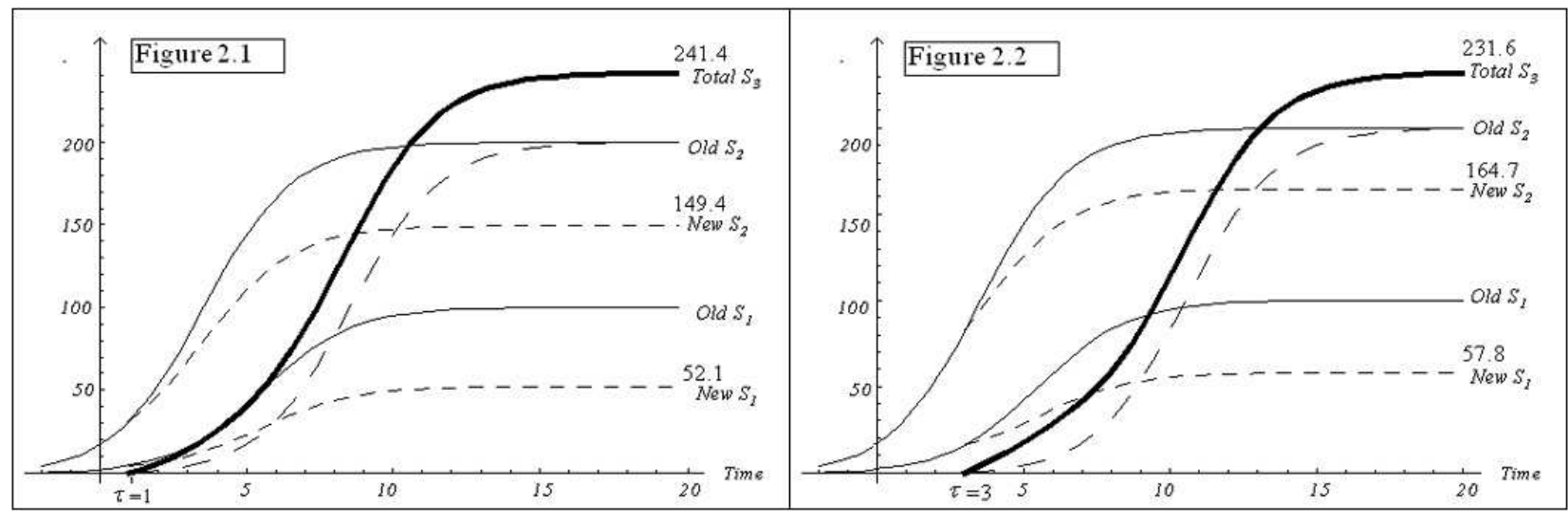

Figure 2. Examples of Cumulative Sales. 

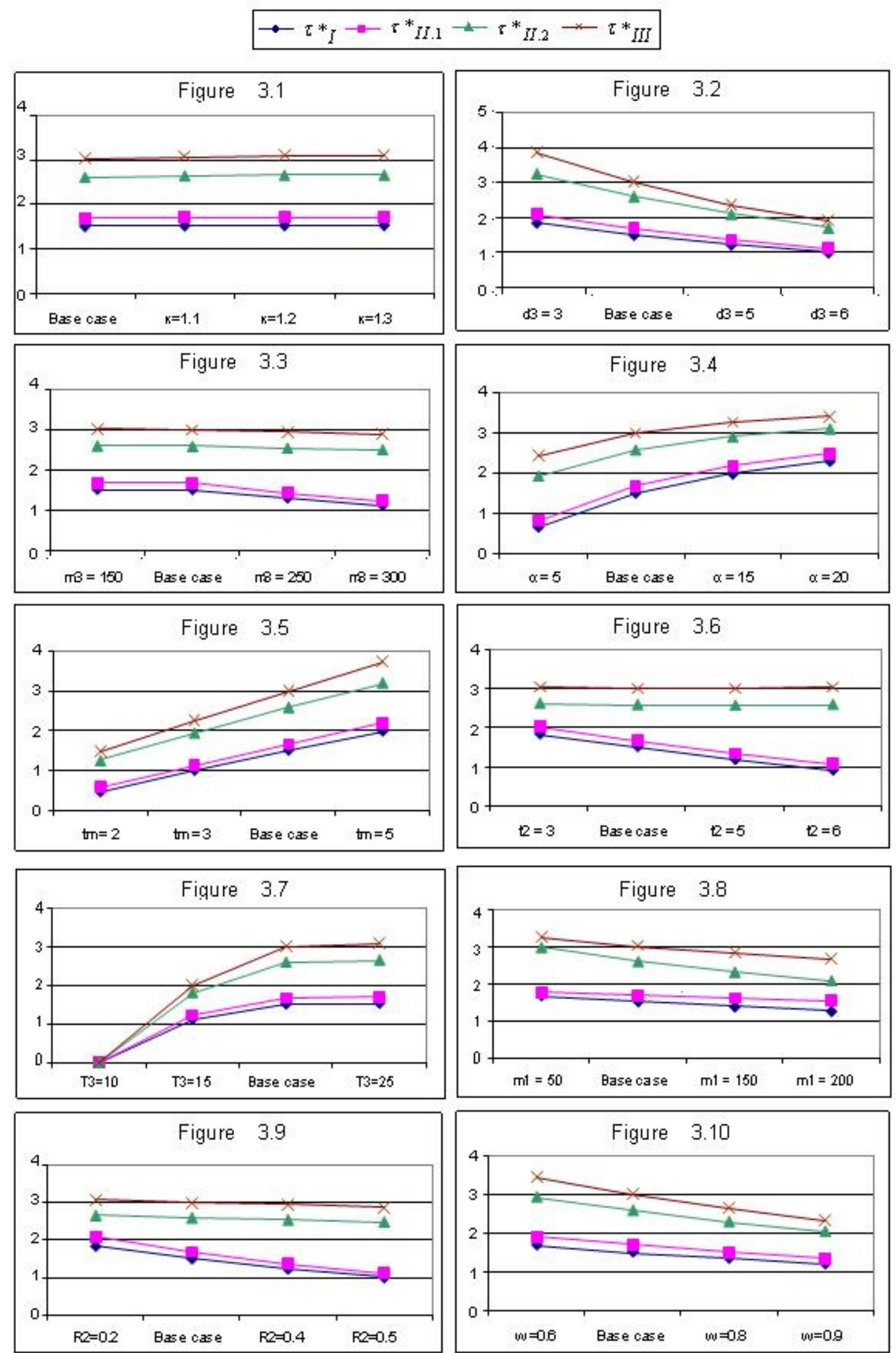

Figure 3. Optimal launch time of the fusion product versus various factors. 


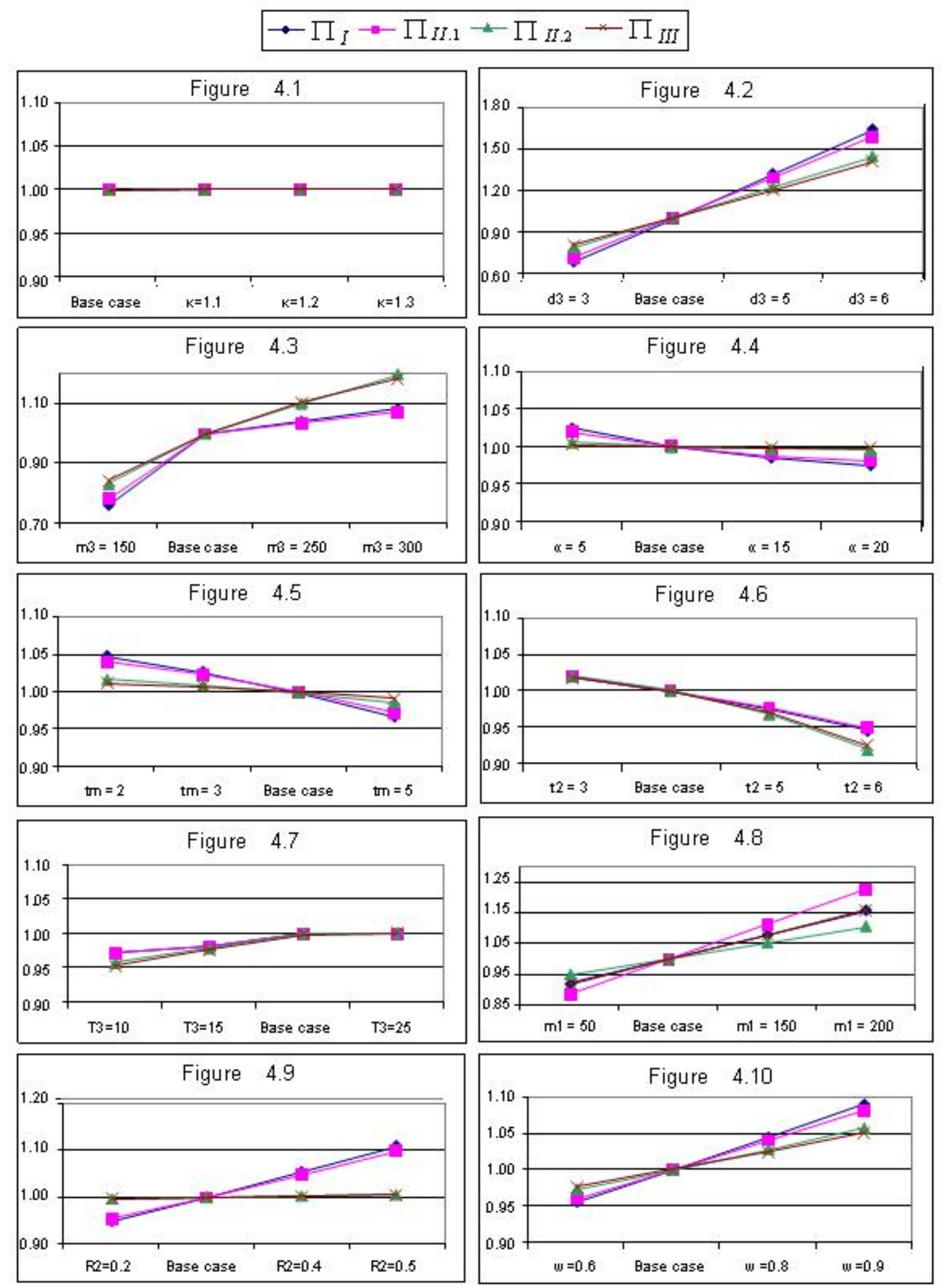

Figure 4. This figure shows the impact of parameter change on the profit. The profit of the base case is standardized as 1 and contrasted by the profits of other cases. 